Article

\title{
Circulatory Inflammatory Mediators in the Prediction of Anti-Tuberculous Drug-Induced Liver Injury Using RUCAM for Causality Assessment
}

\author{
Cheng-Maw Ho ${ }^{1}\left(\mathbb{D}\right.$, Chi-Ling Chen ${ }^{2}$, Chia-Hao Chang ${ }^{3 \oplus}$, Meng-Rui Lee ${ }^{3,4}{ }^{(}$, Jann-Yuan Wang ${ }^{3,4, * \mathbb{D}}$, \\ Rey-Heng $\mathrm{Hu}^{1}$ and Po-Huang Lee ${ }^{1}$
}

1 Department of Surgery, National Taiwan University Hospital and College of Medicine, Taipei 10617, Taiwan; miningho@ntu.edu.tw (C.-M.H.); rhhu@ntu.edu.tw (R.-H.H.); pohuang1115@ntu.edu.tw (P.-H.L.)

2 Graduate Institute of Clinical Medicine, National Taiwan University, Taipei 10617, Taiwan; chlnchen@ntu.edu.tw

3 Department of Internal Medicine, National Taiwan University Hospital, Hsinchu Branch, Hsinchu City 300, Taiwan; ntudcm@gmail.com (C.-H.C.); sheepman1024@gmail.com (M.-R.L.)

4 Department of Internal Medicine, National Taiwan University Hospital and College of Medicine, Taipei 10617, Taiwan

* Correspondence: jywang@ntu.edu.tw; Tel.: +886-22-356-2905; Fax: +886-22-358-2867

check for

updates

Citation: Ho, C.-M.; Chen, C.-L.; Chang, C.-H.; Lee, M.-R.; Wang, J.-Y.; $\mathrm{Hu}, \mathrm{R} .-\mathrm{H} . ;$ Lee, P.-H. Circulatory Inflammatory Mediators in the Prediction of Anti-Tuberculous Drug-Induced Liver Injury Using RUCAM for Causality Assessment. Biomedicines 2021, 9, 891. https:// doi.org/10.3390/biomedicines9080891

Academic Editor: Giovanni Squadrito

Received: 17 July 2021

Accepted: 21 July 2021

Published: 25 July 2021

Publisher's Note: MDPI stays neutral with regard to jurisdictional claims in published maps and institutional affiliations.

Copyright: (c) 2021 by the authors. Licensee MDPI, Basel, Switzerland. This article is an open access article distributed under the terms and conditions of the Creative Commons Attribution (CC BY) license (https:// creativecommons.org/licenses/by/ $4.0 /)$

\begin{abstract}
Background: Anti-tuberculous (TB) medications are common causes of drug-induced liver injury (DILI). Limited data are available on systemic inflammatory mediators as biomarkers for predicting DILI before treatment. We aimed to select predictive markers among potential candidates and to formulate a predictive model of DILI for TB patients. Methods: Adult active TB patients from a prospective cohort were enrolled, and all participants received standard anti-tuberculous treatment. Development of DILI, defined as $\geq 5 \times$ ULN for alanine transaminase or $\geq 2.6 \times$ ULN of total bilirubin with causality assessment (RUCAM, Roussel Uclaf causality assessment method), was regularly monitored. Pre-treatment plasma was assayed for 15 candidates, and a set of risk prediction scores was established using Cox regression and receiver-operating characteristic analyses. Results: A total of $19(7.9 \%)$ in 240 patients developed DILI (including six carriers of hepatitis B virus) following anti-TB treatment. Interleukin (IL)-22 binding protein (BP), interferon gamma-induced protein 1 (IP-10), soluble CD163 (sCD163), IL-6, and CD206 were significant univariable factors associated with DILI development, and the former three were backward selected as multivariable factors, with adjusted hazards of $0.20(0.07-0.58), 3.71$ (1.35-10.21), and 3.28 (1.07-10.06), respectively. A score set composed of IL-22BP, IP-10, and SCD163 had an improved area under the curve of $0.744(p<0.001)$. Conclusions: Pre-treatment IL-22BP was a protective biomarker against DILI development under anti-TB treatment, and a score set by additional risk factors of IP-10 and sCD163 employed an adequate DILI prediction.
\end{abstract}

Keywords: drug-induced liver injury; hepatotoxicity; inflammatory mediators; prediction model; tuberculosis; RUCAM (Roussel Uclaf Causality Assessment Method)

\section{Introduction}

Drug-induced liver injury (DILI) is a challenging medical issue because of the obscure nature of liver injury during development and the lack of available biomarkers for early prediction [1]. Ordinarily, liver injury is more often caused by a combination of multiple factors, such as viral hepatitis, alcoholic, or non-alcoholic steatohepatitis, in addition to drugs or complementary medications [2-4]. Prevention is the best policy, although difficult.

Significant concern about developing DILI during anti- tuberculous (TB) treatment has been raised because it can result in the modification or discontinuation of anti-TB treatment in about one-sixth of TB patients [5,6] and is difficult to manage, rendering further dissemination of Mycobacterium tuberculosis. DILI under anti-TB treatment (anti-TB 
DILI) is the most common cause of DILI and drug-induced acute liver failure in many Asian countries [7] and the second most common cause of drug-induced acute on chronic liver failure in Asia [7]. The incidence of anti-TB DILI varies depending on the definition used to define DILI and varies from $3.4 \%$ to $7.3 \%[7,8]$.

Studies of biomarkers in DILI can be applied in diagnosis, severity, or prognosis [9] Currently few biomarkers for DILI (including anti-TB medications) are useful for early detection, monitoring, or for diagnosis purposes [9]. Steuerwald et al. had previously compared serum immune analytes (14 cytokines, 7 chemokines, and 6 growth factors) at DILI onset and after 6 months, with healthy controls, among the USA DILI cohort and demonstrated that high levels of expression of cytokines associated with innate immunity are associated with a poor prognosis, whereas high levels of expression of adaptive cytokines are associated with good long-term prognosis and eventual recovery [10] However, their cohort is heterogeneous and not specific to TB medications. In addition, they used the serum of patients who had already developed DILI.

Risk factor analysis of circulating inflammatory mediators, particularly for TB patients, is not adequately addressed, if ever, that would greatly bridge the gap between genetic and epidemiological risk. We hypothesise that pre-treatment plasma biomarkers are associated with the development of DILI in patients with TB. We aimed to analyse selective cytokines, chemokines, growth factors, and regulatory proteins, and relevant collected clinical data to formulate a prediction model of DILI once TB treatment started.

\section{Patients and Methods}

\subsection{Patients}

Adult patients (aged between 20 and 90 years) who were diagnosed with cultureconfirmed all-susceptible pulmonary $\mathrm{TB}$, joined previous prospective clinical studies conducted at the National Taiwan University Hospital (NTUH) (Institutional Review Board [IRB] No.: 201303063RINC, 201403048RIND, 201512169RINA) and agreed to share residual pre-treatment blood samples for other later studies. This study was approved by the NTUH IRB (202005100RINC). Among the participants in this study cohort, published data were derived from only fifty-three patients enrolled in IRB No. 201512169RINA in a non-relevant subject [11].

\subsection{Protocol}

Before anti-tuberculous treatment began, aspartate transaminase (AST), alanine transaminase (ALT), total bilirubin (T-Bil), and serological tests for hepatitis B virus (HBV), hepatitis $\mathrm{C}$ virus (HCV), and human immunodeficiency virus (HIV) were performed. A liver function test (LFT) was then performed at 2, 4, 6, 8, 12, and 16 weeks after the start of anti-tuberculous treatment or whenever symptoms of hepatitis developed, and clinically relevant hepatitis was suspected by the primary care physician during the course of treatment [12] Additional testing would be performed if primary care physicians suggested based on clinical condition.

All participants received standard anti-tuberculosis treatment of daily isoniazid (INH), rifampin (RMP), ethambutol (EMB), and pyrazinamide (PZA) in the first two months and daily INH and RIF for the subsequent four months. The regimen was modified, if necessary, by the primary care physician [12].

\subsection{Clinical Parameters}

Demographic and clinical data, including underlying comorbidities (presence of hepatitis $A$ virus (HAV), hepatitis B virus (HBV), hepatitis $C$ virus $(\mathrm{HCV})$, hepatitis $E$ virus (HEV), human immunodeficiency virus (HIV), diabetes mellitus (DM), hypertension, positive cancer history, autoimmune disease, and receiving haemodialysis), chronic smoking and alcohol use, date of blood sampling and starting anti-TB treatment, and blood test results were collected. 


\subsection{Outcome Definitions of DILI}

Baseline abnormal liver function test results were defined as elevated ALT $\geq 2$ times the upper limit of normal (ULN) before the commencement of anti-TB therapy. When abnormal liver function panels were noted during follow-up, patients were screened for viral hepatitis (HAV, $\mathrm{HEV}$, and reactivation of $\mathrm{HBV})$, autoimmune hepatitis and enquiry on the use of other hepatotoxic drugs or complementary and alternative medicine [7]. DILI is defined as $\geq 5 \times$ ULN for ALT or $\geq 2.6 \times$ ULN of total bilirubin, based on the Division of AIDS (DAIDS) Table for Grading the Severity of Adult and Paediatric Adverse Events [13] and causality assessment by using RUCAM (Roussel Uclaf Causality Assessment Method) [14].

\subsection{Assays of Plasma Biomarkers}

Inflammatory markers in plasma potentially relevant to TB-associated DILI were assayed using the Invitrogen ${ }^{\mathrm{TM}}$ ProcartaPlex ${ }^{\mathrm{TM}}$ Human 65-plex panel (Thermo Fisher Scientific, Waltham, MA, USA) kit and enzyme-linked immunosorbent assay (ELISA), including cytokines (IL-22, IL-22 binding protein (IL-22BP), IL-6, IL-10, IL-12p70, IL-17A, IL-23, IP-10 (CXCL10, interferon gamma (IFN-gamma)-induced protein 10)], chemokines [RANTES (CCL5, regulated on activation, normal T cell expressed and secreted), MIG (CXCL9, monokine induced by gamma interferon), MIP (macrophage inflammatory proteins)-1 $\beta$ ], macrophage activation markers (CD206 and sCD163), and growth factors (fibroblast growth factor [FGF]-2, platelet-derived growth factor [PDGF]-bb), according to the manufacturers' recommendations (ELISA kits for IL-22BP, CD206, and sCD163: MyBioSource, San Diego, CA, USA; for other markers: Thermo Fisher Scientific, Waltham, MA, USA).

\subsection{Statistical Analysis}

Data are expressed as mean \pm standard deviation, median (interquartile range [IQR]), or number (percentage) when appropriate. Student's $t$-test, the $\chi^{2}$ test, Fisher's exact test, or the Mann-Whitney U test were used, where appropriate, for the comparison of variables. Cox's proportional hazard model was used for univariable and stepwise multivariable analyses; the latter was employed to identify prognostic factors associated with the development of DILI during anti-TB treatment and to adjust for potential confounding factors.

Cytokine levels were transformed into binary variables according to Youden's index [15] before entering the Cox regression analysis. Optimal cut-off values for biomarkers were calculated from a receiver operating characteristic (ROC) curve. Tertiles (three 3levels) of biomarkers, with cut-off values determined from the non-DILI group, were used in the sensitivity analysis.

Statistical significance was set at $p<0.05$. Analyses were performed using the Statistical Package for Social Sciences (SPSS) ${ }^{\circledR}$ version 21.0 (SPSS Inc., Chicago, IL, USA).

\section{Results}

\subsection{Demographics, DILI Correlation, and DILI-Free Survival}

The patient flow is shown in Figure 1. From December 2011 to July 2017, 1442 patients were diagnosed with culture-confirmed pulmonary TB. Among the 363 patients who agreed to participate, a total of 240 patients were enrolled in this study after excluding patients who were transferred out later, had drug-resistant $\mathrm{TB}$, and refused to share residual blood samples.

Patient demographics are listed in Table 1. None of the patients had hepatic tuberculosis, HIV, or chronic alcohol use. Nineteen patients (7.9\%) met the criteria of DILI (DILI group) (17 hepatocellular type, 1 cholestatic type, and 1 mixed type) during anti-TB treatment, with 3 being judged as highly probable and 16 as probable according to the RUCAM score and hepatologist consultation (Table 2). Most patients (18/19, 94.7\%) developed DILI within 70 days of initiating anti-TB treatment. DILI-free survival for 1, 2, 3, 6, and 9 months were $98.7 \%, 94.5 \%, 92.7 \%, 92.0 \%$, and $90.4 \%$, respectively. Compared to those without DILI (non-DILI group), the DILI group were older, had more HBV carriers, had fewer mean dosing days for INH and PZA, and higher initial total bilirubin (all $p<0.05)$. 
Clinical factors with trends of frequency difference between the groups included more active smoking, fewer dosing days of EMB, and more abnormal initial liver function tests in the DILI group (all $p<0.10$ ).

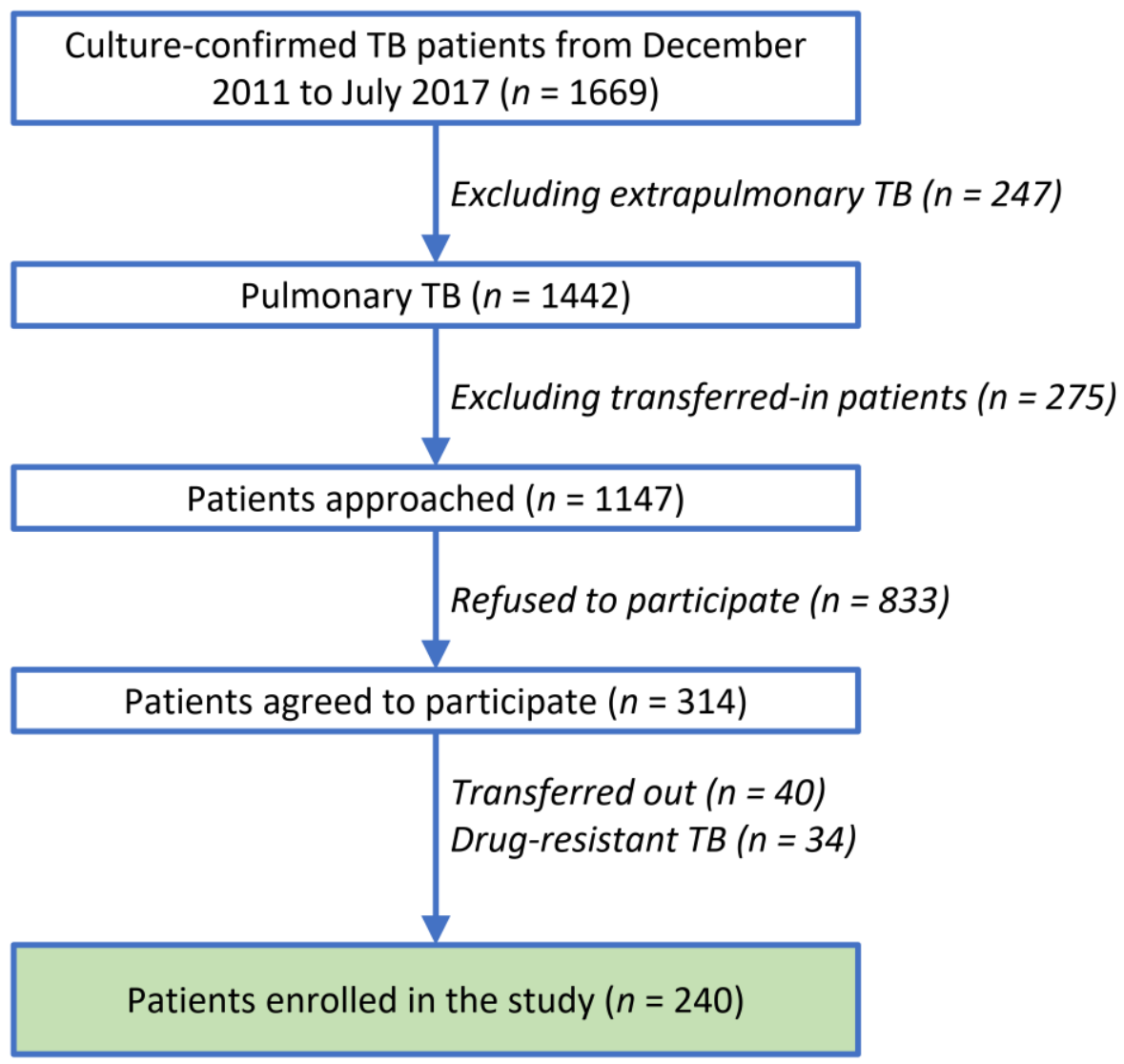

Figure 1. Patient flowchart.

Table 1. Characteristics of patient demographics.

\begin{tabular}{ccccc}
\hline & All $(\boldsymbol{n = 2 4 0 )}$ & DILI $(\boldsymbol{n = 1 9 )}$ & Non-DILI $(\boldsymbol{n = 2 2 1 )}$ & $\boldsymbol{p}$ \\
\hline Age (years) & $55.8 \pm 17.7$ & $63.9 \pm 14.4$ & $55.1 \pm 17.8$ & 0.021 \\
Male sex (\%) & $143(59.6)$ & $12(63.2)$ & $131(59.3)$ & 0.812 \\
BMI $\left(\mathrm{kg} / \mathrm{m}^{2}\right)$ & $21.8 \pm 3.3$ & $21.7 \pm 2.3$ & $21.8 \pm 3.4$ & 0.798 \\
Active smoking & $39(16.3)$ & $6(31.6)$ & $33(14.9)$ & 0.099 \\
Diabetes mellitus & $38(15.8)$ & $3(15.8)$ & $35(15.8)$ & 1.000 \\
Hypertension & $34(14.2)$ & $2(10.5)$ & $32(14.5)$ & 1.000 \\
Presence of cancer history & $42(17.5)$ & $4(21.1)$ & $38(17.2)$ & 0.752 \\
Hepatitis B virus & $31(12.9)$ & $6(31.6)$ & $25(11.3)$ & 0.023 \\
Hepatitis C virus & $4(1.7)$ & $1(5.3)$ & $3(1.4)$ & 0.283 \\
Haemodialysis & $3(1.3)$ & $1(5.3)$ & $2(0.9)$ & 0.220 \\
Autoimmune disease & $10(4.2)$ & $1(5.3)$ & $9(4.1)$ & 0.569 \\
Baseline AST (U/L) & $26.5 \pm 25.1$ & $41.8 \pm 46.7$ & $25.1 \pm 22.1$ & 0.178 \\
Baseline ALT (U/L) & $22.4 \pm 26.4$ & $40.0 \pm 52.0$ & $20.9 \pm 22.7$ & 0.141 \\
Baseline T-Bil (mg/dL) & $0.6 \pm 0.4$ & $1.2 \pm 1.1$ & $0.6 \pm 0.3$ & 0.047 \\
Abnormal baseline LFT & $7(2.9)$ & $2(10.5)$ & $5(2.3)$ & 0.098 \\
\hline
\end{tabular}

Abbreviations: ALT, alanine transaminase; AST, aspartic transaminase; BMI, body mass index; CI, confidence interval; DILI, drug-induced liver injury; HR, hazards ratio; LFT, liver function test; T-Bil, total bilirubin; Data were either mean \pm standard deviation or number $(\%)$. 
Table 2. Initial and peak liver profile as well as the Roussel Uclaf Causality Assessment Method (RUCAM) score of the 19 cases with drug-induced liver injury (DILI).

\begin{tabular}{|c|c|c|c|c|c|c|c|c|}
\hline \multirow[b]{2}{*}{ Case } & \multicolumn{2}{|c|}{ Initial Data } & \multirow{2}{*}{$\begin{array}{l}\text { Time to } \\
\text { Peak } \\
\text { (Days) }\end{array}$} & \multicolumn{2}{|c|}{ Peak Data } & \multirow[b]{2}{*}{ DILI Type } & \multirow[b]{2}{*}{$\begin{array}{l}\text { RUCAM } \\
\text { Score }\end{array}$} & \multirow[b]{2}{*}{$\begin{array}{c}\text { Probability } \\
\text { of DILI }\end{array}$} \\
\hline & $\mathrm{ALT} \geq 2 \mathrm{ULN}$ & $\mathrm{Bil}-\mathrm{T} \geq 2 \mathrm{mg} / \mathrm{dL}$ & & $\begin{array}{c}\text { ALT } \\
(\mathrm{ULN})\end{array}$ & $\begin{array}{c}\text { Bil-T } \\
\text { (mg/dL) }\end{array}$ & & & \\
\hline $92.6 / F$ & 0 & 0 & 33 & 8.8 & 1.2 & hepatocellular & 6 & probable \\
\hline $47.8 / \mathrm{M}$ & 0 & 0 & 32 & 18.3 & 1.0 & hepatocellular & 7 & probable \\
\hline $65.1 / \mathrm{M}$ & 0 & 0 & 37 & 20.8 & 0.9 & hepatocellular & 8 & probable \\
\hline $48.7 / \mathrm{F}^{*}$ & 0 & 0 & 38 & 10.9 & 0.5 & hepatocellular & 6 & probable \\
\hline $72.4 / \mathrm{F}$ & 0 & 0 & 50 & 8.6 & 0.6 & hepatocellular & 7 & probable \\
\hline $60.4 / F$ & 0 & 0 & 24 & 5.6 & 0.3 & hepatocellular & 8 & probable \\
\hline $65.5 / \mathrm{F}^{*}$ & 0 & 0 & 54 & 15.1 & 1.3 & hepatocellular & 8 & probable \\
\hline $47.7 / \mathrm{F}$ & 0 & 0 & 42 & 13.7 & 2.1 & hepatocellular & 8 & probable \\
\hline $52.9 / \mathrm{M}^{*}$ & 0 & 0 & 35 & 7.0 & 0.9 & hepatocellular & 8 & probable \\
\hline $66.3 / M^{*}$ & 0 & 1 & 64 & 3.6 & 4.4 & cholestatic & 7 & probable \\
\hline $60.1 / \mathrm{M}$ & 1 & 0 & 55 & 7.3 & 1.3 & hepatocellular & 10 & $\begin{array}{l}\text { highly } \\
\text { probable }\end{array}$ \\
\hline $64.1 / \mathrm{M}$ & 0 & 0 & 55 & 5.4 & 0.5 & hepatocellular & 6 & probable \\
\hline $91.0 / \mathrm{M}$ & 0 & 0 & 25 & 1.8 & 5.0 & mixed & 8 & probable \\
\hline $38.8 / \mathrm{M}^{*}$ & 0 & 0 & 48 & 6.3 & 0.7 & hepatocellular & 6 & probable \\
\hline $85.5 / \mathrm{M}$ & 1 & 1 & 6 & 55.8 & 4.1 & hepatocellular & 9 & $\begin{array}{l}\text { highly } \\
\text { probable }\end{array}$ \\
\hline $58.5 / \mathrm{M}^{*}$ & 0 & 0 & 70 & 21.8 & 0.7 & hepatocellular & 6 & probable \\
\hline $59.5 / \mathrm{M}$ & 0 & 0 & 68 & 8.0 & 0.8 & hepatocellular & 6 & probable \\
\hline $70.0 / \mathrm{F}$ & 0 & 0 & 74 & 9.8 & 0.7 & hepatocellular & 12 & $\begin{array}{l}\text { highly } \\
\text { probable }\end{array}$ \\
\hline $66.6 / \mathrm{M}$ & 0 & 0 & 35 & 25.1 & 0.8 & hepatocellular & 8 & probable \\
\hline
\end{tabular}

Abbreviations: ALT, alanine transaminase; Bil-T, total bilirubin; ULN, upper limit of normal; ${ }^{*}$ hepatitis B virus carrier.

In univariable Cox analysis, age, with a hazard ratio (HR) of 1.03, (95\% confidence interval [95\% CI], 1.00-1.06), HBV carriers, with an HR of 3.29, (1.25-8.78), and baseline T-Bil, with an HR of 3.46, (2.27-5.30) were clinical risk factors associated with developing DILI after starting TB treatment.

\subsection{Plasma Biomarkers and DILI Correlation}

The plasma levels of the 15 candidate biomarkers are shown in Figure 2. The DILI group had significantly higher levels of IL-6 and IP-10 than the non-DILI group. The optimal cut-off values for these biomarkers, with individual sensitivity and specificity, are displayed in Table S1.

In univariable Cox analysis, IL-6 (HR, 2.49 (1.00-6.20)), IP-10 (3.21 (1.22-8.44)), CD206 (4.02 (1.17-13.8)), and sCD163 (3.47 (1.15-10.5)) were significant risk factors associated with DILI development (Table 3). IL-22BP (0.32 [0.11-0.88]) was a significant protective factor against the development of DILI. In multivariable Cox analysis, IL-22BP, IP-10, and sCD163 were independent factors associated with DILI development, with respective adjusted HRs of $0.20(0.07-0.58), 3.71$ (1.35-10.21), and 3.28 (1.07-10.06) (Table 3).

\subsection{IP-10, IL22BP, and SCD163: Patient Characteristics, DILI-Free Survivals, and Sensitivity Analysis}

The point of ROC curve maximizing the Youden Index (sum of sensitivity and specificity) was determined as the optimal cut-off value [16]. The cut-off values were $3.9 \mathrm{pg} / \mathrm{mL}$, $520 \mathrm{ng} / \mathrm{L}$, and $1.32 \mathrm{ng} / \mathrm{mL}$ for IP-10, IL22BP, and sCD163, respectively. Patient characteristics, stratified by the respective dichotomous levels of IP-10, IL-22BP, and sCD163, are shown in Table 4 . Patients with high IP-10 levels $(n=96)$ were older, had underlying hypertension, and the hepatitis $C$ virus, and had higher levels of baseline LFT (AST, ALT, and T-Bil) than those with lower IP-10 levels (all $p<0.05$ ) (Table 4). Compared to low levels, for patients with high IL-22BP levels $(n=27)$, more underlying hypertension and less HBV infection were observed; for higher sCD163 levels $(n=19)$, older patients were 
observed (all $p<0.05)$. Patients with high IP-10, low IL-22BP, and high sCD163 levels had significantly inferior individual DILI-free survival than those in the other groups (Figure 3, upper panel). These trends could be seen in tertiles (Figure 3, middle panel) and the HBV subgroup (Figure 3, lower panel).

\subsection{Scoring Predicting DILI}

An arbitrary scoring system was developed by the summation of dichotomous levels of IP-10, IL-22BP, and sCD163. Namely, the score $=$ IP-10 (0 or 1) + IL-22BP (0 (high) or 1 (low) $+\mathrm{sCD} 163$ (0 or 1). DILI-free survival stratified by three categories of scores $(0,1$, and $\geq 2)$ is shown in Figure 4A, with higher scores indicating a greater risk of DILI development. The HR for score 1 (score 0 as reference) was 5.15 (1.11-23.80), and for score $\geq 2,11.26$ (1.90-66.72) after adjusting age and HBV (model 1, Figure 4B). When further adjusting baseline total bilirubin level (model 2), the similar trend was demonstrated with the HR $4.23(0.85-21.0)$ for score 1 and 14.45 (2.17-96.12) for score 2. The AUC based on the scoring system was $0.744(p<0.001)$, higher than that of IL-22BP, IP-10, and sCD163 and 12 other inflammatory markers listed in Table S1.
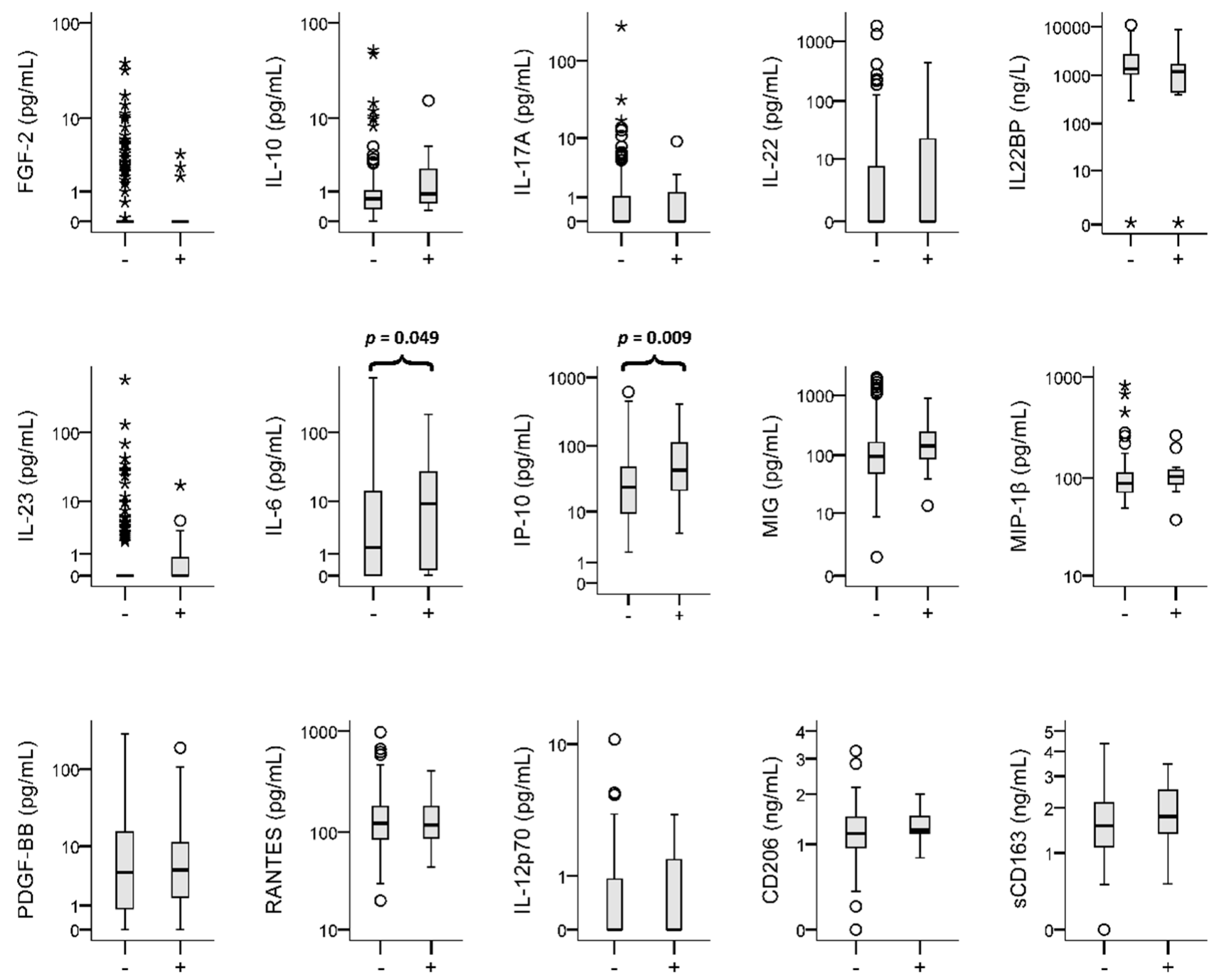

Figure 2. Box plots for cytokines. The box plot visualizes the following summary statistics: the middle line represents the median; the lower hinge corresponds to the first quartile (25th); the upper hinge corresponds to the third quartile (75th); upper and lower whiskers extend from the hinge respectively to the largest value and smallest value or no further than $1.5 \times$ interquartile range; data beyond the whiskers are outliers (circles) and extremes (asterisks). Outliers are at least 1.5 box lengths from the median and extremes are at least three box lengths from the median. 
Table 3. Univariable and multivariable analyses of categorical cytokine risk factors for predicting DILI development in TB patients.

\begin{tabular}{ccccccc}
\hline & \multicolumn{3}{c}{ Univariable } & \multicolumn{3}{c}{ Multivariable Backward Selection } \\
\cline { 2 - 6 } & HR & 95CI & $p$ & HR & 95\% CI & $p$ \\
\hline IL-10 & 6.32 & $(0.84-47.5)$ & 0.073 & & & \\
IL-17A & 1.22 & $(0.47-3.22)$ & 0.683 & & & \\
IL-22 & 1.68 & $(0.67-4.17)$ & 0.267 & & & \\
IL-22BP & 0.32 & $(0.11-0.88)$ & 0.027 & 0.20 & & \\
IL-23 & 1.40 & $(0.51-3.90)$ & 0.515 & & & \\
IL-6 & 2.49 & $(1.00-6.20)$ & 0.049 & & & \\
IP-10 & 3.21 & $(1.22-8.44)$ & 0.018 & 3.71 & $(1.35-10.21)$ & \\
MIG & 1.82 & $(0.53-6.25)$ & 0.342 & & & \\
MIP-1b & 5.43 & $(0.73-40.7)$ & 0.100 & 6.81 & $(0.87-53.47)$ & \\
PDGF-BB & 1.62 & $(0.47-5.57)$ & 0.443 & & & \\
RANTES & 0.61 & $(0.08-4.54)$ & 0.626 & & & \\
IL-12p70 & 2.12 & $(0.83-5.38)$ & 0.115 & & & \\
CD206 & 4.02 & $(1.17-13.8)$ & 0.027 & & & \\
sCD163 & 3.47 & $(1.15-10.5)$ & 0.028 & 3.28 & $(1.07-10.06)$ & \\
\hline
\end{tabular}

Abbreviations: CI, confidence interval; DILI, drug-induced liver injury; HR, hazards ratio; IL, interleukin; IL-22BP IL-22 binding protein; IP-10, interferon gamma-induced protein 10; MIG, monokine induced by interferongamma; MIP-1b, macrophage inflammatory protein-1beta; PDGF-BB, platelet-derived growth factor-BB; RANTES, Regulated upon Activation, Normal T Cell Expressed and Presumably Secreted; sCD163, soluble CD163; TB, tuberculosis.
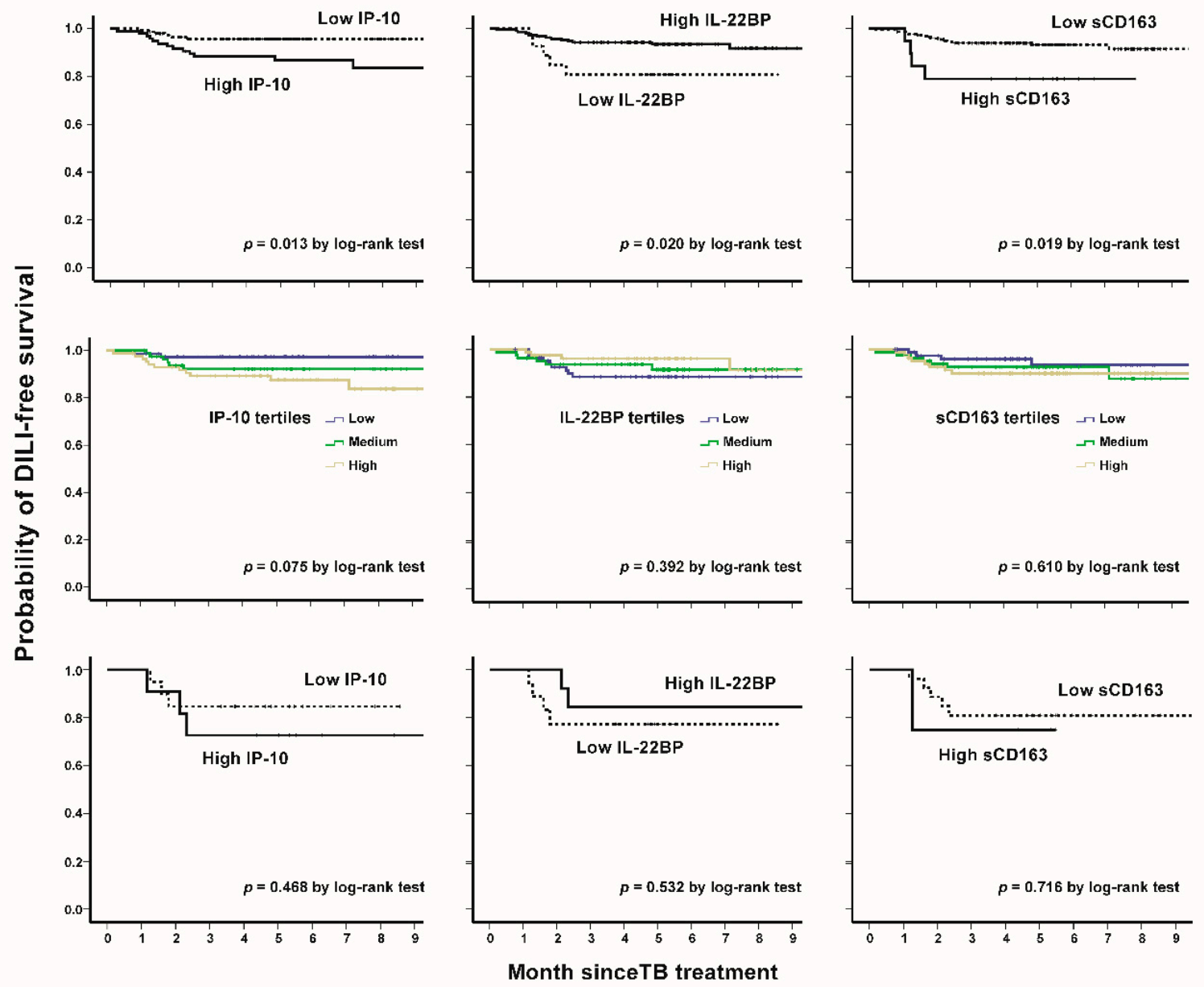

Figure 3. DILI-free survival curves, stratified by IP-10, IL-22BP, and sCD163 levels (upper panel); in tertiles (middle panel), and in the HBV subgroup (lower panel), respectively. 
Table 4. Patient characteristics, stratified by IP-10, IL-22 BP, and sCD163 levels.

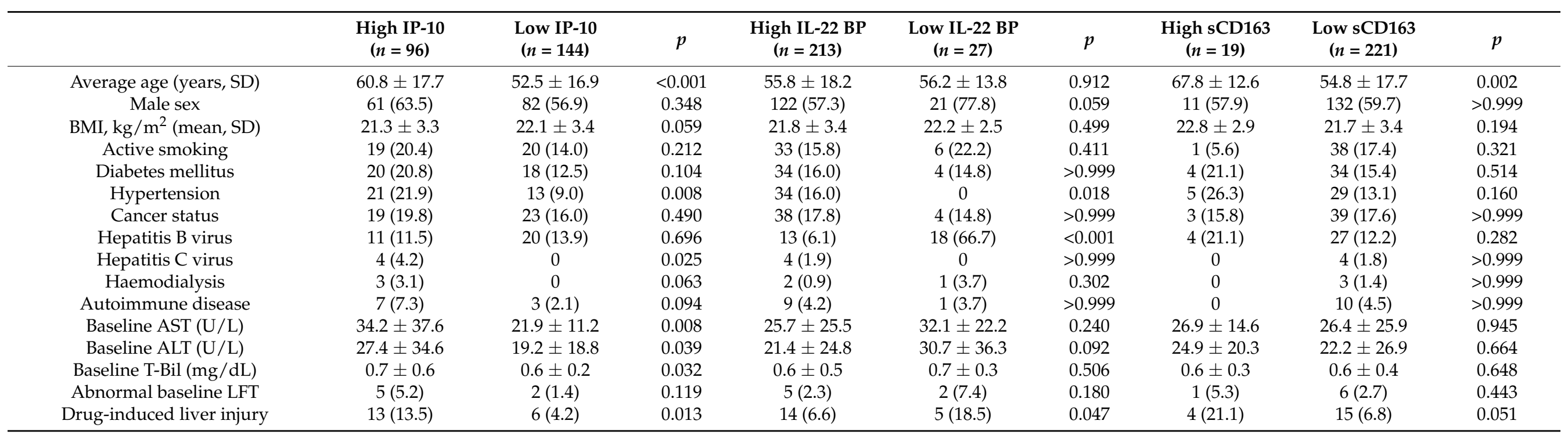

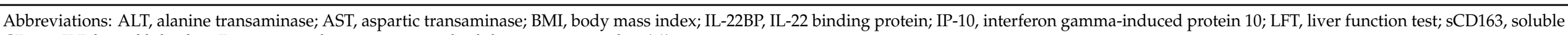
CD163; T-Bil, total bilirubin; Data were either mean \pm standard deviation or number (\%). 


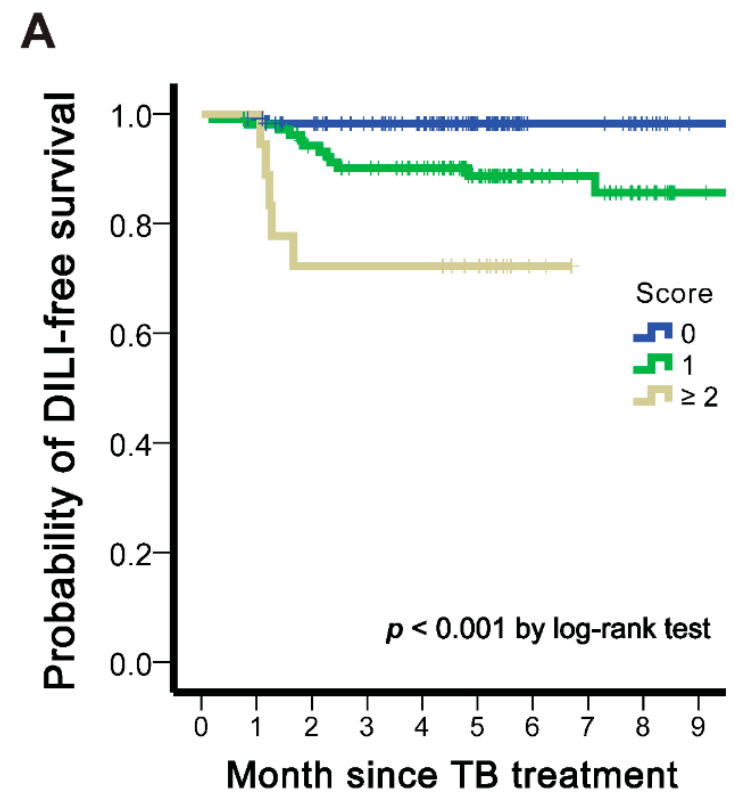

B

\begin{tabular}{ccccccc}
\hline & \multicolumn{3}{c}{ Model 1 } & \multicolumn{3}{c}{ Model 2 } \\
\cline { 2 - 6 } & HR & 95CI & $\boldsymbol{p}$ & HR & 95CI & $p$ \\
\hline Score & & & 0.029 & & & 0.021 \\
1 vs. 0 & 5.15 & $1.11-23.80$ & 0.036 & 4.23 & $0.85-21.0$ & 0.078 \\
$\geq 2$ vs. 0 & 11.26 & $1.90-66.72$ & 0.008 & 14.45 & $2.17-96.12$ & 0.006 \\
Age & 1.02 & $0.99-1.05$ & 0.212 & 1.02 & $0.98-1.05$ & 0.393 \\
HBV & 2.02 & $0.71-5.78$ & 0.190 & 1.46 & $0.39-5.46$ & 0.572 \\
Baseline T-Bil & - & - & - & 5.12 & $1.56-16.75$ & 0.007 \\
\hline
\end{tabular}

Figure 4. DILI-free survival in score stratification. (A) Kaplan Meier plot and (B) adjusted hazard ratios in cox regression analysis.

\section{Discussion}

Our study revealed four main findings. First, nearly $8 \%$ of patients with cultureconfirmed drug-susceptible pulmonary TB developed DILI after treatment, and over $90 \%$ of them occurred within 70 days. Second, advanced age and HBV carriers were two clinical risk factors for DILI development in our prospective cohort. Third, among the 15 plasma biomarker candidates, pre-treatment IP-10, sCD163, and IL-22BP were narrowed down as risk (IP-10 and sCD163) and protective (IL-22BP) predictors of future DILI. Lastly, after adjusting for age and HBV status, a score composed of the three aforementioned biomarkers had a dose-responsive hazard for DILI prediction and employed an AUC of 0.744 .

We validated the 19 cases of DILI ( 3 as highly probable and 16 as probable) cautiously by using RUCAM and hepatologist consultation. Many DILI cases would not be DILI but something else if the cases were not evaluated for causality assessment method such as RUCAM [14] Rathi et al. had used RUCAM prospectively in their paper and identified 40 patients with anti-TB DILI, among all 82 patients with DILI after excluding eight patients for whom DILI was deemed unlikely to be responsible for liver injury [17] RUCAM was appreciated in more than 80,000 DILI cases published worldwide [18]. Education and training on RUCAM should be encouraged to improve the results of the studies and the dayto-day work in pharmacovigilance departments in companies or in regulatory agencies [19]. It is also expected to improve RUCAM with biomarkers or other criteria provided that the 
validation process replaces expert opinion by robust standards such as those used for the original method [19]. However, RUCAM was not designed for suspected chronic DILI, which is mostly an unrecognized preexisting liver disease [20]. Besides, RUCAM was also not designed when a suspected injury occurs on preexisting liver diseases (such as 6 in our 19 cases), a complex condition where expert hepatologists are required [20].

It is possible to reduce treatment-related complications and morbidity by conducting frequent check-ups for liver function in those with a high risk of DILI and prevent further dissemination of TB transmission due to treatment interruption. A net score combining IP-10, IL-22BP, and SCD163 (although the cut-off points were selected statistically without definite priori biological reasoning), developed in our study, appears to help predict the inflammation perturbation caused by TB medications which predispose TB patients to DILI development. Circulating soluble mediators in TB patients before treatment can potentially reflect the immune-inflammatory state in a particular host. We identified a novel protective (IL-22BP) and two harmful (IP-10 and sCD163) roles associated with DILI development. IP-10, secreted by monocytes, endothelial cells, and fibroblasts in response to IFN- $\gamma$ [21], is up-regulated in numerous chronic diseases, including hepatitis B/C [22,23] tuberculosis [24], diabetes [25], and autoimmune disorders [26]. Serum IP-10 level had been reported to be associated with the severity of DILI [27]. probably through the downstream T cell-mediated hepatitis [28]. SCD163 may be released from tissue macrophages and monocytes by a metalloprotease-dependent pathway associated with the inflammation-inducible enzyme TNF- $\alpha$ converting enzyme (TACE/ADAM17) [29]. The exact physiological role as an inflammatory mediator is not clear, although some mechanistic studies have suggested inhibition of activated T lymphocyte proliferation [30] and enhancement of pathogen recognition and phagocytosis [31]. Increased plasma levels of sCD163 have been linked to states of low-grade inflammation such as diabetes, obesity, liver disease, tuberculosis, and atherosclerosis [32-36]. In chronic HBV and HCV infection, sCD163 levels increase with incrementing stages of liver inflammation and fibrosis [37] and the highest levels of sCD163 had been described in patients with acute liver failure especially among patients with fatal outcome [38]. Thus, a correlation between sCD163 level and liver disease severity is evident [39]. In summary, both IP-10 and sCD163 are markers associated with TB and liver inflammation and the inclusion of adaptive (IP-10) and innate (sCD163) immunity in the score set is biologically plausible.

The protective role of IL-22BP in DILI is complex. IL-22 is primarily produced at barrier surfaces by $\mathrm{T}$ cells and innate lymphoid cells and is crucial to maintain epithelial integrity [40], potentially through mediating the crosstalk between leucocytes and the epithelia [41,42]. IL-22, a sibling of IL-17 [43], is mainly secreted by Th17 cells (a helper T lymphocyte subset) and an adaptive cytokine with reported dual-natured pro- and antiinflammatory roles in the restitution of normal tissue and physiology after either sterile or non-sterile inflammation [44]. IL-22BP potently inhibits IL-22 biological effects (with much higher affinity than the IL-22 receptor) and is constitutively expressed in secondary lymphoid organs, breast, and epithelial tissues, preventing exaggerated effects (pathological inflammation) of IL-22 [40]. Also involved in TB and liver disease, IL-22 inhibits intracellular growth of Mycobacterium tuberculosis in human monocyte-derived macrophages [45] and is associated with acetaminophen-related hepatotoxicity and resolution of acute-on-chronic liver failure (ACLF) in small animal models [46,47]. Instead, IL-22 BP emerged as a novel marker in our score set. Consistently, in humans, IL-22BP was reported to be protective and in association with the development of and mortality from ACLF [48]. Nonetheless, the protective effect of IL-22BP should be further tested in large clinical studies.

At presentation with DILI, $84 \%$ of the TB patients had isolated elevation of liver enzymes, yet a high rate of survival. Elevation of liver enzymes in conjunction with jaundice (which was not seen in our study) is well established to reflect more severe DILI and a higher risk of adverse outcome [1] than isolated elevation of liver enzymes. Steuerwald et al. analysed serum taken within two weeks of clinical onset in 78 patients with acute DILI, without any description of causative drugs, and proposed that low values 
of four immune analytes (IL-9, IL-17, PDGF-bb, and RANTES) are predictive of early death [10]. In contrast, our study surveyed pre-treatment plasma samples and, therefore, cytokine profiles derived from our study may not be comparable to their findings. Our study results may be considered as a safety biomarker [49] to predict DILI, and whether these findings apply to other causative agents or diseases warrant further investigation.

Study limitations included no discrimination between idiosyncratic and dose-related hepatotoxicity; the nature of the post-Hoc analysis of prospective studies, in which patients were homogeneously selected by excluding clinically difficult cases, which may potentially limit the severity of DILI and render cytokine statistical analysis toward the null; data regarding the presence of slow acetylator phenotype/genotype of NAT2 gene or cytochrome P450 2E1 (CYP2E1) were not available.

In conclusion, we demonstrated a "safety" scoring of pre-treatment plasma biomarkers (IP-10, IL-22BP, and sCD163) predicting early DILI in patients with culture-confirmed pulmonary TB scheduled for TB medications. In contrast to IP-10 and SCD163, increased levels of IL-22BP may be associated with a protective effect against DILI development. For those with a higher risk of DILI, liver function tests should be performed more frequently for early diagnosis and management of DILI.

Supplementary Materials: The following are available online at https:/ /www.mdpi.com/article/10.3 390/biomedicines9080891/s1, Table S1: Receiver operating characteristic (ROC) curve analysis and area under the ROC curve of baseline plasma cytokines in prediction of drug-induced liver injury.

Author Contributions: Study concept and design: C.-M.H., M.-R.L. and J.-Y.W.; acquisition, analysis, or interpretation of data: C.-M.H., C.-L.C., C.-H.C., M.-R.L. and J.-Y.W.; drafting of the manuscript: C.-M.H., C.-H.C. and J.-Y.W.; critical revision of the manuscript for important intellectual content: R.-H.H. and P.-H.L.; statistical analysis: C.-M.H., C.-L.C. and J.-Y.W.; obtained funding: C.-M.H. and J.-Y.W.; administrative, technical, or material support: C.-H.C., M.-R.L. and J.-Y.W.; Study supervision: R.-H.H. and P.-H.L. All authors have read and agreed to the published version of the manuscript.

Funding: This study was funded by the National Taiwan University Hospital (NTUH 110-S4821). The funder played no role in the study design, data analysis, or manuscript drafting.

Institutional Review Board Statement: The study was conducted according to the guidelines of the Declaration of Helsinki, and approved by the Institutional Review Board of National Taiwan University Hospital.

Informed Consent Statement: Patient consent was waived because all agreed to share residual blood samples when being enrolled in previous studies.

Data Availability Statement: The datasets used and analysed during the current study are available from the corresponding author upon reasonable request.

Acknowledgments: We thank the staff of the Eighth Core Lab, Department of Medical Research of National Taiwan University Hospital for technical support.

Conflicts of Interest: The authors declare no conflict of interest.

\begin{abstract}
Abbreviations
ALT, alanine transaminase; AST, aspartic transaminase; AUC, area under the ROC curve; BMI, body mass index; CI, confidence interval; DILI, drug-induced liver injury; DM, diabetes mellitus; EMB, ethambutol; FGF-2, fibroblast growth factor 2; HAV, hepatitis A virus; HBV, hepatitis B virus; HEV, hepatitis E virus; HIV, human immunodeficiency virus; HR, hazard ratio; IFN-gamma, interferon gamma; IL-22BP, interleukin-22 binding protein; INH, isoniazid; IP-10, interferon gamma (IFN)induced protein 10; IQR, interquartile range, IRB, Institutional Review Board; LFT, liver function test; MIG, monokine induced by interferon-gamma; MIP-1b, macrophage inflammatory protein-1beta; PDGF-BB, platelet-derived growth factor-BB; PZA, pyrazinamide; RANTES, Regulated upon Activation, Normal T Cell Expressed and Presumably Secreted; RMP, rifampin; ROC, receiver operating characteristic; RUCAM, Roussel Uclaf Causality Assessment Method; sCD163, soluble CD163; SD,
\end{abstract}


standard deviation; TB, tuberculosis; T-Bil, total bilirubin; ULN, upper limit of normal.

\section{References}

1. Andrade, R.J.; Aithal, G.P.; Björnsson, E.S.; Kaplowitz, N.; Kullak-Ublick, G.A.; Larrey, D.; Karlsen, T.H.; European Association for the Study of the Liver. EASL Clinical Practice Guidelines: Drug-induced liver injury. J. Hepatol. 2019, 70, 1222-1261. [CrossRef] [PubMed]

2. Fuster, D.; Samet, J. Alcohol use in patients with chronic liver disease. N. Engl. J. Med. 2018, 379, 1251-1261. [CrossRef]

3. Wen, C.S.; Ho, C.M. Alcohol or not: A review comparing initial mechanisms, contributing factors, and liver transplantation outcomes between alcoholic and nonalcoholic steatohepatitis. Eur. Med. J. 2018, 3, 40-48.

4. Ho, C.M.; Lee, C.H.; Wang, J.Y.; Lee, P.H.; Lai, H.S.; Hu, R.H. Nationwide longitudinal analysis of acute liver failure in Taiwan. Medicine 2014, 93, e35. [CrossRef]

5. Wang, J.Y.; Liu, C.H.; Hu, F.C.; Chang, H.C.; Liu, J.L.; Chen, J.M.; Yu, C.J.; Lee, L.N.; Kao, J.H.; Yang, P.C. Risk factors of hepatitis during anti-tuberculous treatment and implications of hepatitis virus load. J. Infect. 2011, 62, 448-455. [CrossRef]

6. Wang, J.Y.; Lee, L.N.; Yu, C.J.; Chien, Y.J.; Yang, P.C.; Tami Group. Factors influencing time to smear conversion in patients with smear-positive pulmonary tuberculosis. Respirology 2009, 14, 1012-1019. [CrossRef]

7. Devarbhavi, H.; Aithal, G.; Treeprasertsuk, S.; Takikawa, H.; Mao, Y.; Shasthry, S.M.; Hamid, S.; Tan, S.S.; Philips, C.A.; George, J.; et al. Drug-induced liver injury: Asia Pacific Association of Study of Liver consensus guidelines. Hepatol. Int. 2021, 15, 258-282. [CrossRef]

8. Tweed, C.D.; Wills, G.H.; Crook, A.M.; Dawson, R.; Diacon, A.H.; Louw, C.E.; McHugh, T.D.; Mendel, C.; Meredith, S.; Mohapi, L.; et al. Liver toxicity associated with tuberculosis chemotherapy in the REMoxTB study. BMC Med. 2018, 16, 46. [CrossRef]

9. Meunier, L.; Larrey, D. Drug-induced liver injury: Biomarkers, requirements, candidates, and validation. Front. Pharmacol. 2019, 10, 1482. [CrossRef]

10. Steuerwald, N.M.; Foureau, D.M.; Norton, H.J.; Zhou, J.; Parsons, J.C.; Chalasani, N.; Fontana, R.J.; Watkins, P.B.; Lee, W.M.; Reddy, K.R.; et al. Profiles of serum cytokines in acute drug-induced liver injury and their prognostic significance. PLoS ONE 2013, 8, e81974. [CrossRef]

11. Chang, S.Y.; Chen, M.L.; Lee, M.R.; Liang, Y.C.; Lu, T.P.; Wang, J.Y.; Yan, B.S. SP110 polymorphisms are genetic markers for vulnerability to latent and active tuberculosis infection in Taiwan. Dis. Markers 2018, 2018, 4687380. [CrossRef] [PubMed]

12. Chiang, C.Y. Taiwan Guidelines for TB Diagnosis and Treatment, 6th ed.; Centers for Disease Control, R.O.C.: Taipei, Taiwan, 2018.

13. U.S. Department of Health and Human Services; National Institutes of Health; National Institute of Allergy and Infectious Diseases; Division of AIDS. Division of AIDS (DAIDS) Table for Grading the Severity of Adult and Pediatric Adverse Events, Corrected Version 2.1. 2017. Available online: https://rsc.niaid.nih.gov/clinical-research-sites/daids-adverse-event-gradingtables (accessed on 6 June 2020).

14. Danan, G.; Teschke, R. RUCAM in drug and herb induced liver injury: The update. Int. J. Mol. Sci. 2016, 17, 14. [CrossRef]

15. Schisterman, E.F.; Perkins, N.J.; Liu, A.; Bondell, H. Optimal cut-point and its corresponding Youden Index to discriminate individuals using pooled blood samples. Epidemiology 2005, 16, 73-81. [CrossRef]

16. Fluss, R.; Faraggi, D.; Reiser, B. Estimation of the Youden Index and its associated cutoff point. Biom. J. $2005,47,458-472$. [CrossRef]

17. Rathi, C.; Pipaliya, N.; Patel, R.; Ingle, M.; Phadke, A.; Sawant, P. Drug induced liver injury at a tertiary hospital in india: Etiology, clinical features and predictors of mortality. Ann. Hepatol. 2017, 16, 442-450. [CrossRef]

18. Teschke, R.; Danan, G. Worldwide use of RUCAM for causality assessment in 81,856 idiosyncratic DILI and 14,029 HILI cases published 1993-mid 2020: A comprehensive analysis. Medicines 2020, 7, 62. [CrossRef]

19. Danan, G.; Teschke, R. Roussel Uclaf Causality Assessment Method for drug-induced liver injury: Present and future. Front. Pharmacol. 2019, 10, 853. [CrossRef] [PubMed]

20. Teschke, R.; Danan, G. Prospective Indian study of DILI with confirmed causality using the Roussel Uclaf Causality Assessment Method (RUCAM): A report of excellence. Ann. Hepatol. 2017, 16, 324-325. [CrossRef]

21. Luster, A.D.; Unkeless, J.C.; Ravetch, J.V. Gamma-interferon transcriptionally regulates an early-response gene containing homology to platelet proteins. Nature 1985, 315, 672-676. [CrossRef] [PubMed]

22. Cornberg, M.; Wiegand, S.B. Importance of IP-10 in hepatitis B. Antivir. Ther. 2016, 21, 93-96. [CrossRef]

23. Romero, A.I.; Lagging, M.; Westin, J.; Dhillon, A.P.; Dustin, L.B.; Pawlotsky, J.M.; Neumann, A.U.; Ferrari, C.; Missale, G.; Haagmans, B.L.; et al. Interferon (IFN)-gamma-inducible protein-10: Association with histological results, viral kinetics, and outcome during treatment with pegylated IFN-alpha 2a and ribavirin for chronic hepatitis C virus infection. J. Infect. Dis. 2006, 194, 895-903. [CrossRef]

24. You, E.; Kim, M.H.; Lee, W.I.; Kang, S.Y. Evaluation of IL-2, IL-10, IL-17 and IP-10 as potent discriminative markers for active tuberculosis among pulmonary tuberculosis suspects. Tuberculosis 2016, 99, 100-108. [CrossRef]

25. Antonelli, A.; Ferrari, S.M.; Corrado, A.; Ferrannini, E.; Fallahi, P. CXCR3, CXCL10 and type 1 diabetes. Cytokine Growth Factor Rev. 2014, 25, 57-65. [CrossRef] 
26. Singh, U.P.; Singh, N.P.; Guan, H.; Hegde, V.L.; Price, R.L.; Taub, D.D.; Mishra, M.K.; Nagarkatti, M.; Nagarkatti, P.S. The severity of experimental autoimmune cystitis can be ameliorated by anti-CXCL10 Ab treatment. PLoS ONE 2013, 8, e79751. [CrossRef]

27. Xie, Z.Y.; Chen, E.M.; Ouyang, X.X.; Xu, X.; Ma, S.; Ji, F.; Wu, D.; Zhang, S.; Zhao, Y.; Li, L. Metabolomics and cytokine analysis for identification of severe drug-induced liver injury. J. Proteome Res. 2019, 18, 2514-2524. [CrossRef]

28. Dai, S.; Liu, F.; Qin, Z.; Zhang, J.; Chen, J.; Ding, W.X.; Feng, D.; Ji, Y.; Qin, X. Kupffer cells promote T-cell hepatitis by producing CXCL10 and limiting liver sinusoidal endothelial cell permeability. Theranostics 2020, 10, 7163-7177. [CrossRef]

29. Etzerodt, A.; Berg, R.M.; Plovsing, R.R.; Andersen, M.N.; Bebien, M.; Habbeddine, M.; Lawrence, T.; Møller, H.J.; Moestrup, S.K. Soluble ectodomain CD163 and extracellular vesicle-associated CD163 are two differently regulated forms of 'soluble CD163' in plasma. Sci. Rep. 2017, 7, 40286. [CrossRef]

30. Högger, P.; Sorg, C. Soluble CD163 inhibits phorbol ester-induced lymphocyte proliferation. Biochem. Biophys. Res. Commun. 2001, 288, 841-843. [CrossRef]

31. Kneidl, J.; Löffler, B.; Erat, M.C.; Kalinka, J.; Peters, G.; Roth, J.; Barczyk, K. Soluble CD163 promotes recognition, phagocytosis and killing of Staphylococcus aureus via binding of specific fibronectin peptides. Cell Microbiol. 2012, 14, 914-936. [CrossRef]

32. Parkner, T.; Sorensen, L.P.; Nielsen, A.R.; Fischer, C.P.; Bibby, B.M.; Nielsen, S.; Pedersen, B.K.; Møller, H.J. Soluble CD163: A biomarker linking macrophages and insulin resistance. Diabetologia 2012, 55, 1856-1862. [CrossRef] [PubMed]

33. Zanni, M.V.; Burdo, T.H.; Makimura, H.; Williams, K.C.; Grinspoon, S.K. Relationship between monocyte/macrophage activation marker soluble CD163 and insulin resistance in obese and normal-weight subjects. Clin. Endocrinol 2012, 77, 385-390. [CrossRef]

34. Gronbaek, H.; Sandahl, T.D.; Mortensen, C.; Vilstrup, H.; Møller, H.J.; Møller, S. Soluble CD163, a marker of Kupffer cell activation, is related to portal hypertension in patients with liver cirrhosis. Aliment. Pharmacol. Ther. 2012, 36, 173-180. [CrossRef]

35. Hong, J.Y.; Lee, H.J.; Kim, S.Y.; Chung, K.S.; Kim, E.Y.; Jung, J.Y.; Park, M.S.; Kim, Y.S.; Kim, S.K.; Chang, J.; et al. Efficacy of IP-10 as a biomarker for monitoring tuberculosis treatment. J. Infect. 2014, 68, 252-258. [CrossRef]

36. Aristoteli, L.P.; Møller, H.J.; Bailey, B.; Moestrup, S.K.; Kritharides, L. The monocytic lineage specific soluble CD163 is a plasma marker of coronary atherosclerosis. Atherosclerosis 2006, 184, 342-347. [CrossRef]

37. Kazankov, K.; Barrera, F.; Møller, H.J.; Bibby, B.M.; Vilstrup, H.; George, J.; Grønbaek, H. Soluble CD163, a macrophage activation marker, is independently associated with fibrosis in patients with chronic viral hepatitis B and C. Hepatology 2014, 60, 521-530. [CrossRef]

38. Møller, H.J.; Grønbaek, H.; Schiødt, F.V.; Holland-Fischer, P.; Schilsky, M.; Munoz, S.; Hassanein, T.; Lee, W.M.; U.S. Acute Liver Failure Study Group. Soluble CD163 from activated macrophages predicts mortality in acute liver failure. J. Hepatol. 2007, 47, 671-676. [CrossRef] [PubMed]

39. Nielsen, M.C.; Hvidbjerg Gantzel, R.; Trebicka, J.; Møller, H.J.; Grønbæk, H. Macrophage activation markers, CD163 and CD206, in acute-on-chronic liver failure. Cells 2020, 9, 1175. [CrossRef] [PubMed]

40. Martin, J.C.; Bériou, G.; Heslan, M.; Chauvin, C.; Utriainen, L.; Aumeunier, A.; Scott, C.L.; Mowat, A.; Cerovic, V.; Houston, S.A.; et al. Interleukin-22 binding protein (IL-22BP) is constitutively expressed by a subset of conventional dendritic cells and is strongly induced by retinoic acid. Mucosal Immunol. 2014, 7, 101-113. [CrossRef]

41. Zheng, Y.; Valdez, P.A.; Danilenko, D.M.; Hu, Y.; Sa, S.M.; Gong, Q.; Abbas, A.R.; Modrusan, Z.; Ghilardi, N.; de Sauvage, F.J.; et al. Interleukin-22 mediates early host defense against attaching and effacing bacterial pathogens. Nat. Med. 2008, 14, 282-289. [CrossRef]

42. Aujla, S.J.; Chan, Y.R.; Zheng, M.; Fei, M.; Askew, D.J.; Pociask, D.A.; Reinhart, T.A.; McAllister, F.; Edeal, J.; Gaus, K.; et al. IL-22 mediates mucosal host defense against Gram-negative bacterial pneumonia. Nat. Med. 2008, 14, 275-281. [CrossRef]

43. Eyerich, S.; Eyerich, K.; Cavani, A.; Schmidt-Weber, C. IL-17 and IL-22: Siblings, not twins. Trends Immunol. 2010, 31, 354-361. [CrossRef]

44. Alabbas, S.Y.; Begun, J.; Florin, T.H.; Oancea, I. The role of IL-22 in the resolution of sterile and nonsterile inflammation. Clin. Transl. Immunol. 2018, 7, e1017. [CrossRef] [PubMed]

45. Dhiman, R.; Venkatasubramanian, S.; Paidipally, P.; Barnes, P.F.; Tvinnereim, A.; Vankayalapati, R. Interleukin 22 inhibits intracellular growth of Mycobacterium tuberculosis by enhancing calgranulin A expression. J. Infect. Dis 2014, 209 , 578-587. [CrossRef]

46. Feng, D.; Wang, Y.; Wang, H.; Weng, H.; Kong, X.; Martin-Murphy, B.V.; Li, Y.; Park, O.; Dooley, S.; Ju, C.; et al. Acute and chronic effects of IL-22 on acetaminophen-induced liver injury. J. Immunol. 2014, 193, 2512-2518. [CrossRef]

47. Xiang, X.; Feng, D.; Hwang, S.; Ren, T.; Wang, X.; Trojnar, E.; Matyas, C.; Mo, R.; Shang, D.; He, Y.; et al. Interleukin-22 ameliorates acute-on-chronic liver failure by reprogramming impaired regeneration pathways in mice. J. Hepatol. 2020, 72, 736-745. [CrossRef]

48. Schwarzkopf, K.; Rüschenbaum, S.; Barat, S.; Cai, C.; Mücke, M.M.; Fitting, D.; Weigert, A.; Brüne, B.; Zeuzem, S.; Welsch, C.; et al. IL-22 and IL-22-binding protein are associated with development of and mortality from acute-on-chronic liver failure. Hepatol. Commun. 2019, 3, 392-405. [CrossRef]

49. Laverty, H.G.; Antoine, D.J.; Benson, C.; Chaponda, M.; Williams, D.; Kevin Park, B. The potential of cytokines as safety biomarkers for drug-induced liver injury. Eur. J. Clin. Pharmacol. 2010, 66, 961-976. [CrossRef] 